\title{
Analysis of Steady Wear State of the Drum Brake
}

\author{
István Páczelt' ${ }^{1}$ Attila Baksa1 ${ }^{1}$ Zenon Mróz ${ }^{2}$ \\ ${ }^{1}$ Institute of Applied Mechanics, University of Miskolc, Miskolc, Hungary \\ ${ }^{2}$ Institute of Fundamental Technological Research, Warsaw, Poland \\ Email: mechpacz@uni-miskolc.hu,mechab@uni-miskolc.hu,zmroz@ippt.pan.pl
}

How to cite this paper: Páczelt, I., Baksa, A. and Mróz, Z. (2020) Analysis of Steady Wear State of the Drum Brake. Open Access Library Journal, 7: e6432.

https://doi.org/10.4236/oalib.1106432

Received: May 15, 2020

Accepted: June 19, 2020

Published: June 22, 2020

Copyright (C) 2020 by author(s) and Open Access Library Inc.

This work is licensed under the Creative Commons Attribution International License (CC BY 4.0).

http://creativecommons.org/licenses/by/4.0/

\section{(c) (i) Open Access}

\begin{abstract}
In the previous paper [1], the formula of the contact pressure distribution in the steady wear state for drum brake system was derived. The contact pressure distributions on the leading and trailing shoes were found to be different and larger on the leading shoe. Using these contact pressure distributions, it is easy way to calculate the braking torque. Assuming the varying position of the shoe pin, the optimization problem can be formulated by requiring the optimal pin position corresponding to maximal braking torque. The steady wear states and optimal designs were specified for both shoes. The elastic displacement field of the drum brake at the optimal contact pressure distribution is calculated by the finite element system ABAQUS. At given rigid body rotation of the shoe, the wear in the leading and trailing shoes in the steady wear state can be easily found.
\end{abstract}

\section{Subject Areas}

Mechanical Engineering

\section{Keywords}

Steady Wear, Drum Brake, Mechanical Contact, Optimization

\section{Introduction}

The analytical expressions of contact pressure distribution in drum brakes were proposed in some papers, starting from the assumptions of Koessler [2]. He expressed the distribution of the normal pressure between drum and brake linings in terms of cosine functions of angular position. The assumed distribution is valid only when the curvatures of the brake lining and the drum are equal. However, the radius of the brake lining varies during operation due to wear process. 
In the paper by Rao and Cao [3], the same assumption was used for the pressure distribution. In this paper, the optimization of the drum brake mechanical system was based on the interval analysis.

A two-dimensional model of the brake shoe was developed by Huang and Shyr [4] in the analysis divided into two parts related to different materials, the metal shoe and the lining plate. The displacement and the traction force at the center point of the constant strain element were used to simplify the integration. The brake drum was assumed as rigid. The effects of the length of lining plate, its initial and final positions, the values of Young moduli were analyzed. The distribution of contact pressure was found different from a cosine curve.

A complete three-dimensional structure of the drum brake has been treated by Hohmann et al. [5] with finite element program ADINA-IN. It was found that the contact pressure over the leading and trailing shoes is different (larger over the leading shoe). Each part of the mechanical system (the break drum, the leading shoe and the trailing shoe) was assumed as elastic. Watson and Newcomb [6] argued that it is more expensive to use the finite element program in the main frame and that the pressure distribution on the lining plate does not vary significantly along the axis of the brake.

In the paper by Ahmed et al. [7] for the analysis of brake system, the finite element program ANSYS was used. The modal analysis and the contact problem were solved. The contact state was determined for different piston loads. At their big value, the contact zone was largest, and for such state, the wear analysis was recommended. However, the wear analysis was not presented in all reviewed papers.

In our analysis, the contact pressure distribution is derived from the variational principle referred to the steady wear state, by minimizing the wear dissipation power, as has been presented in [1]. The steady state pressure distribution is then analytically expressed in terms of sine function of angular position. In our former works [8] [9], some examples were presented illustrating application of the steady wear state variational principle to the analysis of thermo-mechanical wear processes for translating or rotating punches. In [10], the effect of friction anisotropy related to asperity morphology was accounted for in the wear analysis, indicating importance of asperity evolution during the wear process. The finite element analysis presented in this paper illustrates transition from transient to steady wear state and confirms the analytical prediction of contact pressure distribution. The optimal location of shoe rotation pins is now based on the steady state pressure distribution and is aimed at maximizing the brake moment. Good review of structural optimization can be found in [11].

\section{Contact Pressure and Brake Moment at Steady Wear State}

Consider now the drum brake system shown in Figure 1. Two symmetrically located brake shoes are loaded by the forces $F_{0}$ inducing rotation around the axles $\mu p_{n}(\boldsymbol{x})$ and interaction with the rotating drum in the clockwise or anti- 
clockwise directions. The thickness of the shoes is $t_{t t}$. Due to symmetry of the brake system only one shoe is presented in Figure 1. Between the drum and shoe the unilateral contact condition is assumed on the surface $S_{c}^{(\alpha)}$ $\left(R_{0}, \alpha_{i} \leq \alpha \leq \alpha_{u}, t_{t t}\right)$.

The following wear rule is assumed in our analysis

$$
\dot{w}_{n}=\beta\left(\tau_{n}\right)^{b}\left\|\dot{\boldsymbol{u}}_{\tau}\right\|^{a}=\beta\left(\mu p_{n}\right)^{b}\left\|\dot{\boldsymbol{u}}_{\tau}\right\|^{a}=\beta\left(\mu p_{n}\right)^{b} v_{r}^{a}=\tilde{\beta} p_{n}^{b} v_{r}^{a},
$$

where $\beta, a, b$ are the wear parameters, $\mu$ is the coefficient of friction, $\tilde{\beta}=\beta \mu^{b}, v_{r}=\left\|\dot{u}_{\tau}\right\|$ is the relative velocity between two bodies.

In the previous paper [1], the minimization of the generalized wear dissipation power was discussed and the coaxiality rule was derived. It provided the contact pressure distribution in a steady wear state. Introducing the wear rate vector, it was shown that in the steady wear state the wear rate vector is coaxial with the rigid body wear velocity of shoe, which is allowed to execute rigid body motion. Introducing the unit vector specifying orientation of the rigid body velocity on the contact surface

$$
\boldsymbol{e}_{R}=\frac{\dot{\lambda}_{F}+\dot{\lambda}_{M} \times \Delta \boldsymbol{r}}{\left\|\dot{\lambda}_{F}+\dot{\lambda}_{M} \times \Delta \boldsymbol{r}\right\|}
$$

the optimality rule is expressed as follows

$$
\dot{\boldsymbol{w}}_{1}=-\dot{w}_{1, R} \boldsymbol{e}_{R}, \dot{\boldsymbol{w}}_{2}=\dot{w}_{2, R} \boldsymbol{e}_{R},
$$

where $\dot{\lambda}_{F}$ and $\dot{\lambda}_{M}$ are the rigid body relative translation and rotation velocities induced by wear; $\Delta \boldsymbol{r}$ is the position vector.

The total wear rate vector can be decomposed components into normal and tangential directions, thus are

$$
\dot{\boldsymbol{w}}_{n}=\dot{\boldsymbol{w}}_{1, n}+\dot{\boldsymbol{w}}_{2, n}, \quad \dot{\boldsymbol{w}}_{\tau}=\dot{\boldsymbol{w}}_{1, \tau}+\dot{\boldsymbol{w}}_{2, \tau} .
$$

The normal and tangential wear rate components now are

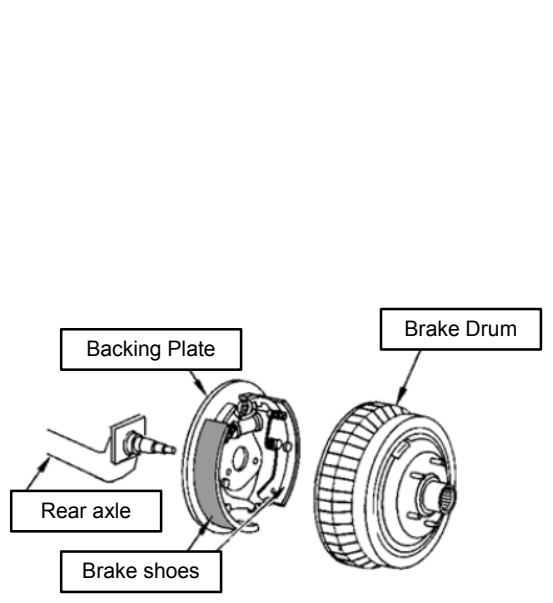

(a)

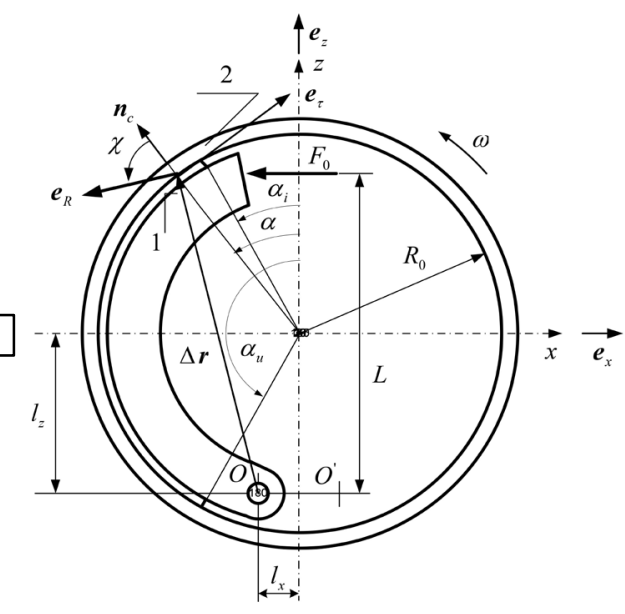

(b)

Figure 1. Drum brake system. (a) Drum brake, (b) geometry and loading of drum brake shoes. 


$$
\dot{w}_{n}=\dot{w}_{R} \cos \chi, \dot{w}_{\tau}=\dot{w}_{R} \sin \chi=\dot{w}_{n} \tan \chi,
$$

here $\chi$ is the angle between $\boldsymbol{n}_{c}$ and $\boldsymbol{e}_{R}$.

The contact pressure $p_{n}(\boldsymbol{x})$ and the friction induced shear stress $\tau_{n}=\mu p_{n}(\boldsymbol{x})$ must satisfy the global equilibrium conditions for the body $B_{1}$.

The contact traction of the drum brake now is expressed as follows

$$
\begin{aligned}
\boldsymbol{t} & =\boldsymbol{t}_{1}^{c}=-\boldsymbol{t}_{2}^{c}=-p_{n} \boldsymbol{\rho}_{c}=-p_{n}\left(\boldsymbol{n}_{c} \pm \mu \boldsymbol{e}_{\tau}\right) \\
& =-p_{n}\left[\left(-\sin \alpha \boldsymbol{e}_{x}+\cos \alpha \boldsymbol{e}_{z}\right) \pm \mu\left(\cos \alpha \boldsymbol{e}_{x}+\sin \alpha \boldsymbol{e}_{z}\right)\right],
\end{aligned}
$$

where the upper sign $(+)$ corresponds to the anticlockwise drum rotation. The geometrical parameters $l_{x}, l_{z}, R_{0}, \alpha$ are shown in Figure 1 . The position vector $\Delta \boldsymbol{r}$ specifying the contact surface with respect to $O$ is

$$
\Delta \boldsymbol{r}=\left(l_{x}-R_{0} \sin \alpha\right) \boldsymbol{e}_{x}+\left(l_{z}+R_{0} \cos \alpha\right) \boldsymbol{e}_{z},
$$

As the shoe is allowed for free rotation, only the moment equilibrium condition of the shoe should be satisfied.

$$
m=m_{c}-M_{0}=0,
$$

where the moment of external load $F_{0}$ is $\boldsymbol{m}_{0}=-F_{0} L \boldsymbol{e}_{y}=-M_{0} \boldsymbol{e}_{y}$, the wear rotation angular velocity equals $\dot{\lambda}_{M}=-\dot{\lambda}_{M} \boldsymbol{e}_{y}$, the moment of contact stress is

$$
\begin{aligned}
& \qquad \begin{aligned}
m_{c} & =\int_{\alpha_{i}}^{\alpha_{u}}\left(\Delta \boldsymbol{r} \times \boldsymbol{t}_{1}^{c}\right) \cdot \boldsymbol{e}_{y} R_{0} t_{t t} \mathrm{~d} \alpha \\
& =\int_{\alpha_{i}}^{\alpha_{u}} p_{n}\left[\left(l_{z} \sin \alpha+l_{x} \cos \alpha\right) \mp \mu\left(l_{z} \cos \alpha-l_{x} \sin \alpha+R_{0}\right)\right] R_{0} t_{t t} \mathrm{~d} \alpha \\
& =\int_{\alpha_{i}}^{\alpha_{u}} p_{n} m^{\mp}(\alpha) R_{0} t_{t t} \mathrm{~d} \alpha
\end{aligned} \\
& \text { where } m^{\mp}(\alpha)=\left[\left(l_{z} \sin \alpha+l_{x} \cos \alpha\right) \mp \mu\left(l_{z} \cos \alpha-l_{x} \sin \alpha+R_{0}\right)\right] .
\end{aligned}
$$

The rigid body wear velocity is coaxial with the unit vector $\boldsymbol{e}_{R}$, so we have

$$
\boldsymbol{e}_{R}=\frac{\Delta \boldsymbol{r} \times \boldsymbol{e}_{y}}{\left|\Delta \boldsymbol{r} \times \boldsymbol{e}_{y}\right|}=\frac{1}{A(\alpha)}\left\{\left(l_{x}-R_{0} \sin \alpha\right) \boldsymbol{e}_{z}-\left(l_{z}+R_{0} \cos \alpha\right) \boldsymbol{e}_{x}\right\},
$$

where

$$
A(\alpha)=\sqrt{\left(l_{x}-R_{0} \sin \alpha\right)^{2}+\left(l_{z}+R_{0} \cos \alpha\right)^{2}} .
$$

Between the vectors $\boldsymbol{n}_{c}$ and $\boldsymbol{e}_{R}$ there is angle $\chi$, its cosine value is

$$
\cos \chi=\boldsymbol{n}_{c} \cdot \boldsymbol{e}_{R}=\left(l_{x} \cos \alpha+l_{z} \sin \alpha\right) \frac{1}{A(\alpha)} .
$$

and the rigid body wear velocity vector equals

$$
\dot{\boldsymbol{w}}_{R}=\dot{w}_{R} \boldsymbol{e}_{R}, \quad \dot{w}_{R}=\frac{\dot{w}_{n}}{\cos \chi} .
$$

Introducing the parameter $c=(b+1) q-1$ and the integral

$$
I_{D_{w}}^{\mp(q)}=\int_{\alpha_{i}}^{\alpha_{u}}\left(m^{\mp}(\alpha)(1 \mp \mu \operatorname{tg} \chi)^{-q}\right)^{1 / c} m^{\mp}(\alpha) R_{0} t_{t t} \mathrm{~d} \alpha,
$$

where the upper sign (-) corresponds to the anticlockwise drum rotation, the lower sign $(+)$ corresponds to clockwise drum rotation. 
In the previous paper [1] the generalized dissipation power was introduced. $D_{w}^{(q)}=\sum_{i=1}^{2}\left(\int_{S_{c}}\left(\boldsymbol{t}_{i}^{c} \cdot \dot{\boldsymbol{w}}_{i}\right)^{q} \mathrm{~d} S\right)^{1 / q}$ with the control parameter $0 \leq q \leq \infty$. The optimal contact pressure and total wear rate for $q=1$ (which corresponds to the steady wear state) were expressed by the following formulae

$$
\begin{gathered}
p_{n}^{\mp}=\frac{F_{0} L}{I_{D_{w}}^{\mp(q=1)}}(A(\alpha) \cos \chi)^{\frac{1}{b}}=\frac{F_{0} L}{I_{D_{w}}^{\mp(q=1)}}\left(l_{x} \cos \alpha+l_{z} \sin \alpha\right)^{1 / b} \\
\dot{w}_{R}^{\mp}=\sum_{i=1}^{2} \tilde{\beta}_{i}\left(\frac{F_{0} L}{I_{D_{w}}^{\mp(q=1)}}\right)^{b}\left(R_{0} \omega\right)^{a_{i}} A(\alpha)
\end{gathered}
$$

or in the other form, which is dependent only on geometrical data and the friction coefficient

$$
\begin{aligned}
p_{n, G}^{\mp} & =\frac{p_{n}^{\mp}}{F_{0} L}=\frac{1}{I_{D_{w}}^{\mp(q=1)}}(A(\alpha) \cos \chi)^{\frac{1}{b}} \\
& =\frac{1}{I_{D_{w}}^{\mp(q=1)}}\left(l_{x} \cos \alpha+l_{z} \sin \alpha\right)^{\frac{1}{b}}\left[1 / \mathrm{mm}^{3}\right]
\end{aligned}
$$

here the dimensions of $I_{D_{w}}^{\mp(q=1)}$ and $p_{n}^{\mp}$ are $\left[\mathrm{mm}^{3+1 / b}\right]$ and $[\mathrm{MPa}]$, of $\dot{w}_{R}^{\mp}$ is $[\mathrm{mm} / \mathrm{s}]$ because $\tilde{\beta}_{i}$ has the dimension $\left[\mathrm{mm}^{1-a+2 b} \cdot \mathrm{s}^{a-1} \cdot \mathrm{N}^{-b}\right]$, which for $a=b=1$ is $\left[\mathrm{mm}^{2} / \mathrm{N}\right]$.

The wear rate $\dot{w}_{R}$ can be expressed directly in terms of the rigid body wear angular velocity $\dot{\lambda}_{M}$

$$
\dot{w}_{R}^{\mp}=\dot{\lambda}_{M}^{\mp} A(\alpha) .
$$

An important result can be stated. The rigid body rotation angular velocity $\dot{\lambda}_{M}^{\mp}$ depends only on the main characteristic of the system and not on local pressure values, thus

$$
\dot{\lambda}_{M}^{\mp}=\sum_{i=1}^{2} \tilde{\beta}\left(\frac{F_{0} L}{I_{D_{w}}^{\mp(q=1)}}\right)^{b}\left(R_{0} \omega\right)^{a_{i}} .
$$

The brake moment in the steady wear state is expressed in terms of the contact pressure (for the optimal contact pressure distribution) by the following integral

$$
M_{T}=M_{\text {brake }}=\int_{\alpha_{i}}^{\alpha_{u}} \mu\left(p_{n}^{-}+p_{n}^{+}\right) R_{0} R_{0} t_{t t} \mathrm{~d} \alpha
$$

or

$$
\frac{M_{\text {brake }}}{2 F_{0} L}=\frac{1}{2} \int_{\alpha_{i}}^{\alpha_{u}} \mu\left(\frac{1}{I_{D_{w}}^{-(q=1)}}+\frac{1}{I_{D_{w}}^{+(q=1)}}\right)\left(l_{x} \cos \alpha+l_{z} \sin \alpha\right)^{1 / b} R_{0}^{2} t_{t t} \mathrm{~d} \alpha
$$

since the drum brake has two shoes. The entire moment from the external load is equal to $2 F_{0} L$, so the relation $\beta_{f}=\frac{M_{\text {brake }}}{2 F_{0} L}$ provides the brake factor, indi- 
cating the portion of the given load moment used in the braking process. The reaction forces in the pin are

$$
\begin{gathered}
F_{x}^{\mp}=\int_{\alpha_{i}}^{\alpha_{u}} p_{n}^{\mp}(\sin \alpha \mp \mu \cos \alpha) R_{0} t_{t t} \mathrm{~d} \alpha-F_{0}, \\
F_{z}^{\mp}=\int_{\alpha_{i}}^{\alpha_{u}} p_{n}^{\mp}(\cos \alpha \pm \mu \sin \alpha) R_{0} t_{t t} \mathrm{~d} \alpha,
\end{gathered}
$$

and their resultant is $F_{r}^{\mp}=\sqrt{\left(F_{x}^{\mp}\right)^{2}+\left(F_{z}^{\mp}\right)^{2}}$. This value is important for the pin design.

In the alternative form, we have

$$
\begin{gathered}
\frac{F_{x}^{\mp}}{F_{0} L}=\frac{1}{F_{0} L} \int_{\alpha_{i}}^{\alpha_{u}} p_{n}^{\mp}(\sin \alpha \mp \mu \cos \alpha) R_{0} t_{t t} \mathrm{~d} \alpha-\frac{1}{L} \\
\frac{F_{z}^{\mp}}{F_{0} L}=\frac{1}{F_{0} L} \int_{\alpha_{i}}^{\alpha_{u}} p_{n}^{\mp}(\cos \alpha \pm \mu \sin \alpha) R_{0} t_{t t} \mathrm{~d} \alpha .
\end{gathered}
$$

\section{Example}

Let us now discuss the numerical results for the assumed wear parameters $a=b=1, \quad \beta_{1}=\beta_{2}=2 \times 10^{-8}, \mu=0.25$, angular velocity of drum $\omega=2.5[1 / \mathrm{sec}]$, geometric parameters $R_{0}=100[\mathrm{~mm}], \quad l_{x}=20[\mathrm{~mm}], \quad l_{z}=80[\mathrm{~mm}]$, $t_{t t}=10[\mathrm{~mm}]$ and loading force $F_{0}=10[\mathrm{kN}]$, which are typically for small break system. Because $D_{w}^{(q)}$ for $q=1$ presents the global wear dissipation power but for $q \rightarrow \infty$ presents its local value, the control parameter $q$ in our calculation is assumed to vary within the interval (0.0 - 10.0). Instead of $q=\infty$ we take $q=10$. In Figure 2, we can see that distribution of $p_{n}$ for $q=2$ and $q=10$ has small difference. Increasing $q$ is unnecessary.

\subsection{Contact Pressure Distribution}

It is seen that the contact pressure and wear rate are localized for the singular value of $q=0.5$. The steady wear state regime corresponds to $q=1$ with the respective contact pressure and wear rate distributions presented in Figures 2-4.

\subsection{Brake Moment}

The brake moment value depends on the pin coordinates $l_{x}, l_{z}$. At fixed geometric values $\left(R_{0}=100[\mathrm{~mm}], t_{t t}=10[\mathrm{~mm}], \alpha_{i}=30^{\circ}, \alpha_{u}=150^{\circ}\right)$, the brake factor value defined as the brake moment to given moment of the load $F_{0}$ : $\beta_{f}=M_{\text {brake }} /\left(2 F_{0} L\right)$ is demonstrated in Figure 5(a). The maximum of the brake factor is reached at $l_{x}=10[\mathrm{~mm}], l_{z}=75[\mathrm{~mm}]$.

The contact zone can be changed by selecting initial and final angles $\alpha_{i}, \alpha_{u}$ and accounting for the constraint of a positive contact pressure, $p_{n} \geq 0$. Requiring that at the end of contact zone there is $l_{x} \cos \alpha_{u}+l_{z} \sin \alpha_{u}=0$, (this point corresponds to a shorter distance from pin, and the pressure at this point can vanish). Using this constraint, we find a larger relative value of the brake 
factor $\beta_{f}=M_{T} /\left(2 F_{0} L\right)$. In Figure 5(b), the dependence of the brake factor $\beta_{f}$ on $l_{x}, l_{z}$ is shown. In Figure 5(c), the function $\alpha_{i}=\alpha_{i}\left(l_{x}, l_{z}\right)$ is presented satisfying the constraint $l_{x} \cos \alpha_{u}+l_{z} \sin \alpha_{u}=0$, where $\alpha_{u}=180-\alpha_{i}$. If we choose the value $\alpha_{i}=\alpha_{i}\left(l_{x}, l_{z}\right)$ higher than the function in Figure $5(\mathrm{c})$, then the brake factor $\beta_{f}$ will be smaller than that of Figure 5(b).
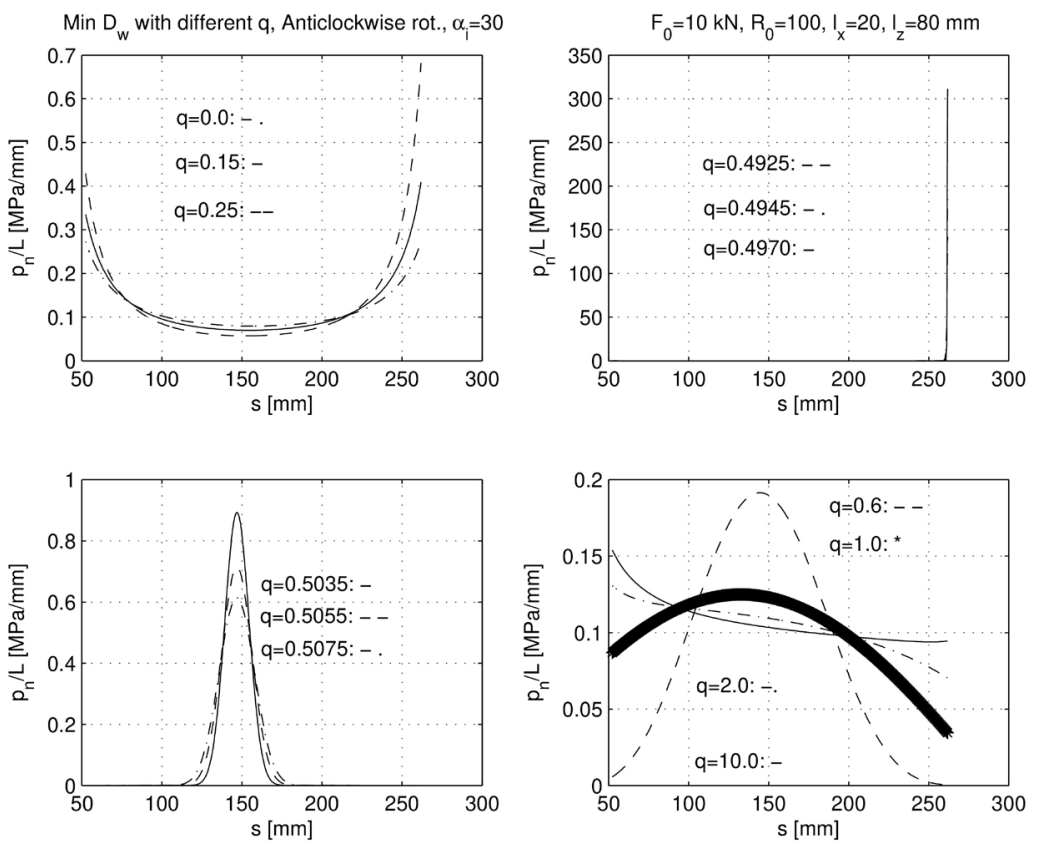

(a)
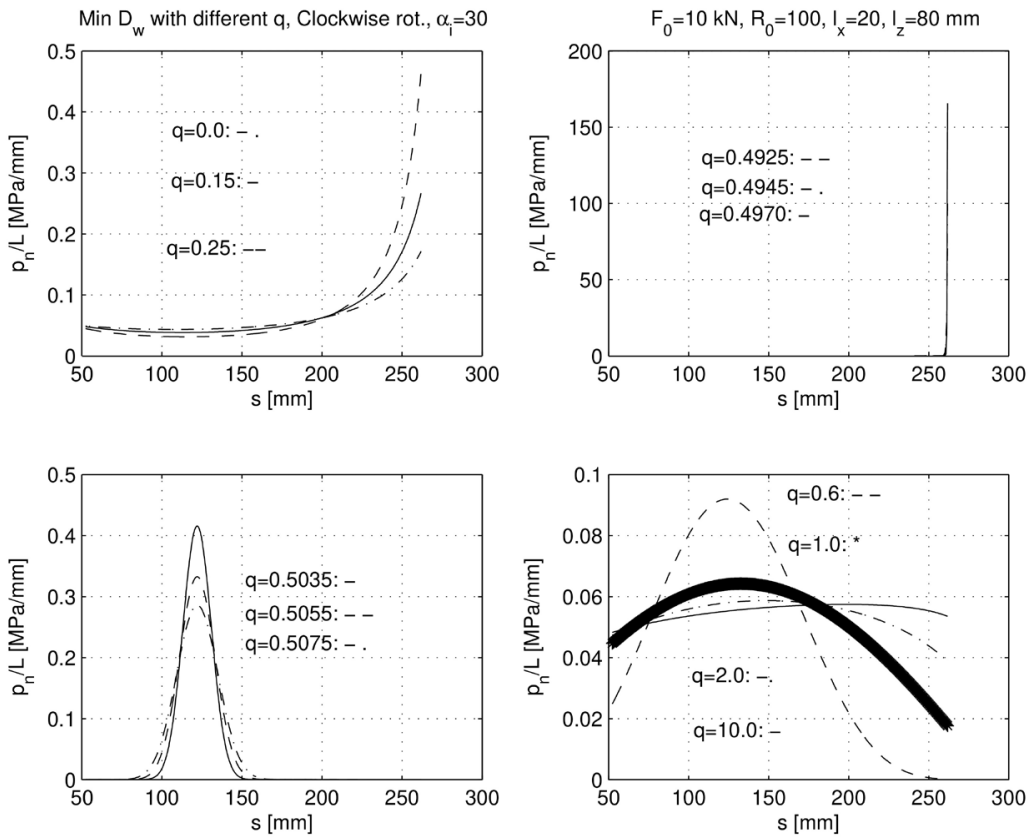

(b)

Figure 2. Optimal contact pressure distribution in drum brake for different values of the control parameter $q$ : (a) anticlockwise drum rotation, (b) clockwise drum rotation. 


\subsection{Wear Profile of the Shoe}

In this section the determination of wear profile in the shoes will be discussed. Figure 6 presents the finite element model of the brake drum. The cylindrical part has the height $t_{c v l}=t_{t t}+2[\mathrm{~mm}]$. In the numerical analysis, Abaqus finite element program with C3D20R type quadratic elements is used. Number of nodes: 99932, degrees of freedom: 299796, number of elements: 19608.
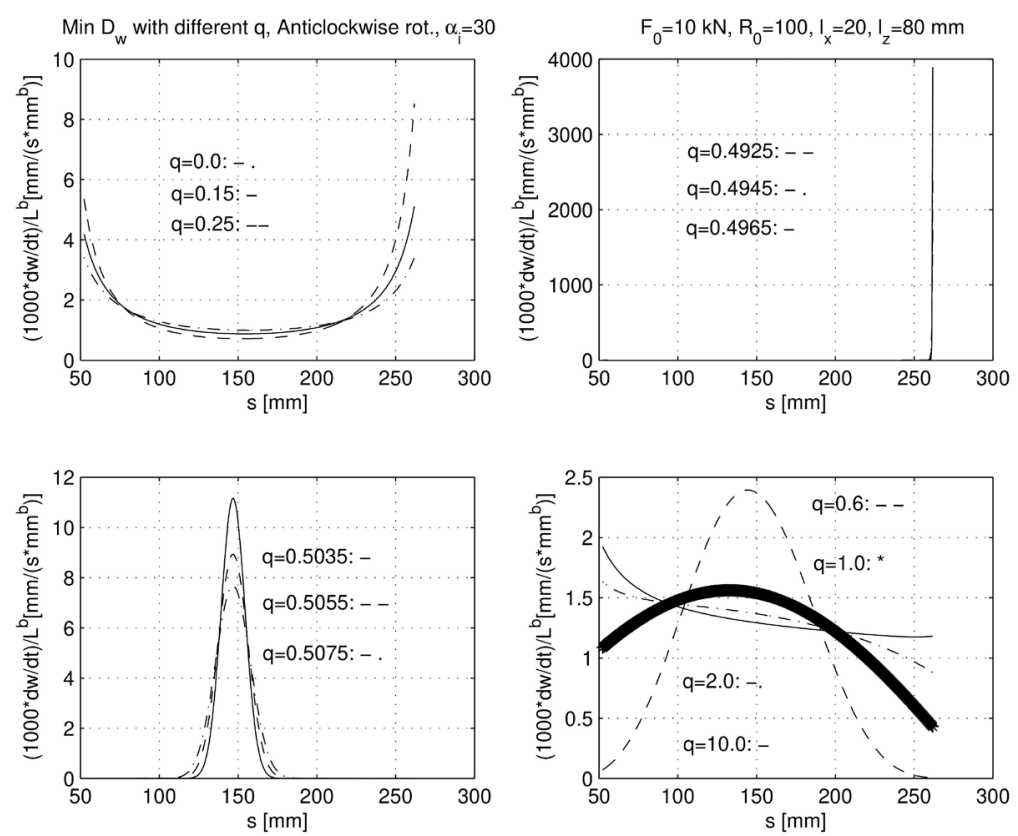

(a)
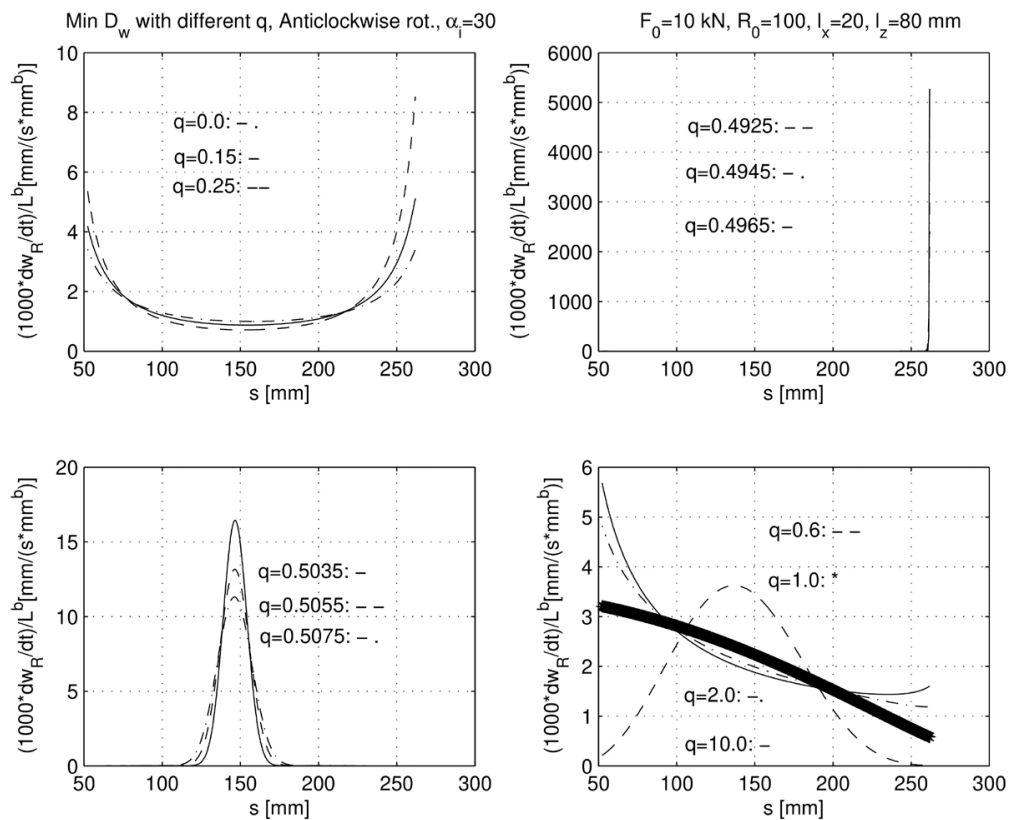

(b)

Figure 3. (a) Normal wear rate distribution $\left(\dot{w}=\dot{w}_{n}\right)$, (b) rigid body wear rate distribution for different values $q$, anticlockwise drum rotation. 


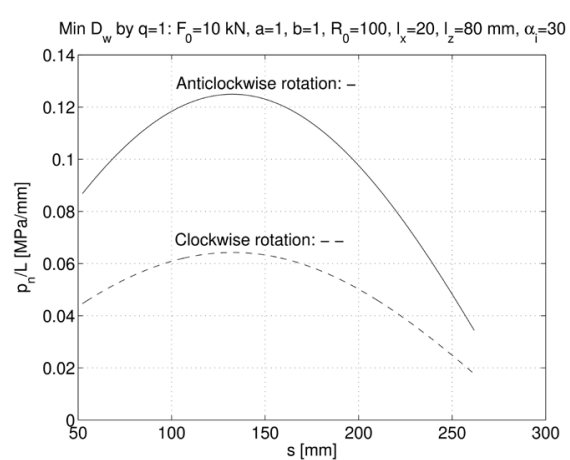

(a)

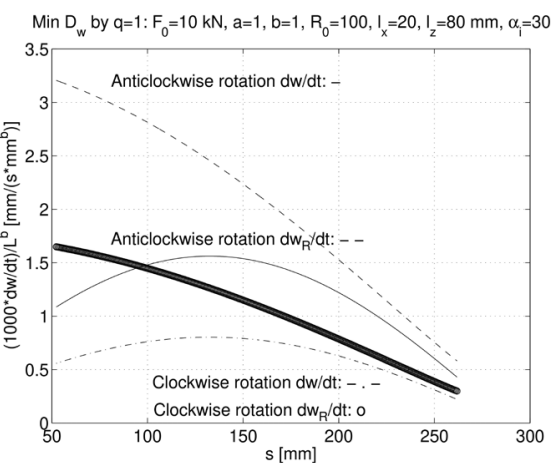

(b)

Figure 4. Contact pressure (a), and wear rate distributions (b), in the steady state: $q=1$.

Drum brake: $F_{0}=10 \mathrm{kN}, R_{0}=100$, alfa $=30$, alfa $=150$

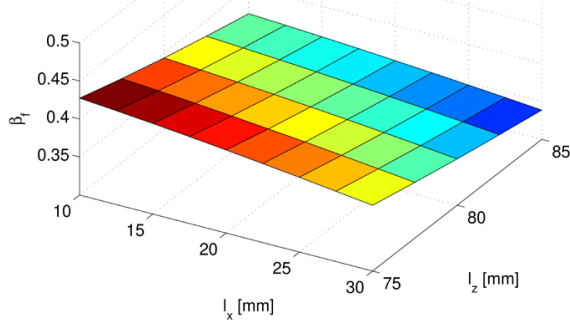

(a)

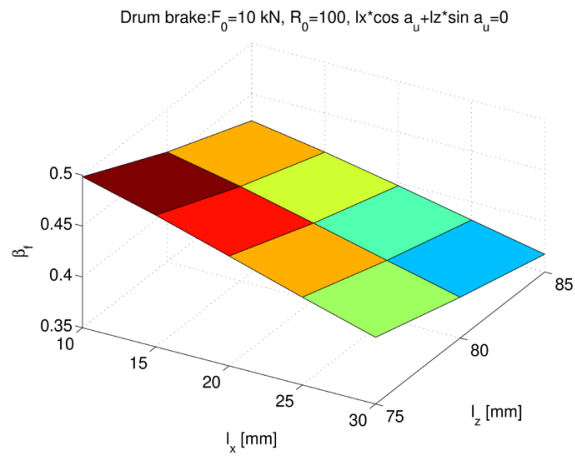

(b)

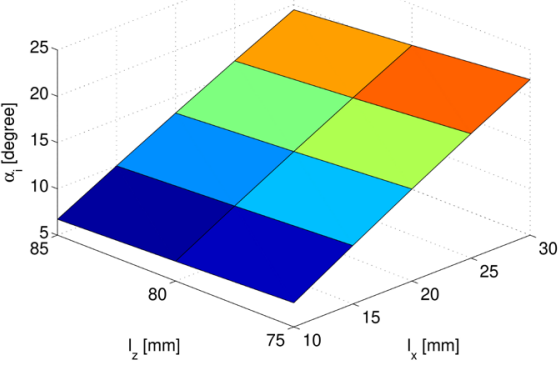

(c)

Figure 5. Brake factor (a) at the fixed $\alpha_{i}, \alpha_{u}$, (b) at the constraint $l_{x} \cos \alpha_{u}+l_{z} \sin \alpha_{u}=0$, (c) the angle function $\alpha_{i}=\alpha_{i}\left(l_{x}, l_{z}\right), \alpha_{u}=180-\alpha_{i}$ satisfying the constraint of case (b).

The surface load on the cylindrical surface of radius $R_{0}$ is given from the steady wear state contact traction (17) at $L=160[\mathrm{~mm}]$ and at load $F_{0}$ that is $p_{\text {surface }}^{\mp}=p_{n}^{\mp}=p_{n, G}^{\mp} F_{0} L \quad[\mathrm{MPa}]$, the shear stress is given by Coulomb law. Using the length coordinate $s$ along the circular profile of radius $R_{0}$ in the clockwise direction the distribution of normal loads $p_{n}^{\mp} t_{t t}[\mathrm{~N} / \mathrm{mm}]$ are demonstrated in Figure 7. The brake drum is rotated in the anticlockwise direction.

After solution of the finite element model, the normal displacement is presented in Figure 8. The displacement is drawn along the different sections $y=1$, $4,7,10,13,16,19[\mathrm{~mm}]$ (increasing in the $-Y$ direction). The curve for section 1 
is upper most at normalized distance 0.25 and the curve for section $7(y=19$ $[\mathrm{mm}])$ is down most at the same distance.
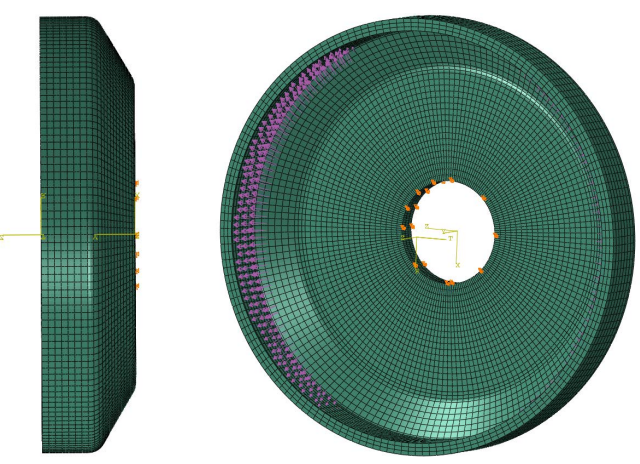

Figure 6. Finite element model of the brake drum. On the central hole perimeter the displacement is assumed to vanish. The load is shown up by arrowhead in the cylinder of radius $R_{0}$.

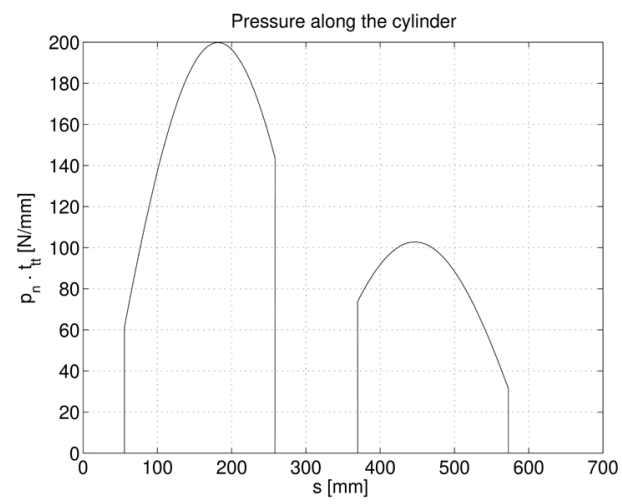

Figure 7. The normal loads in the left (leading) and right (trailing) shoes.

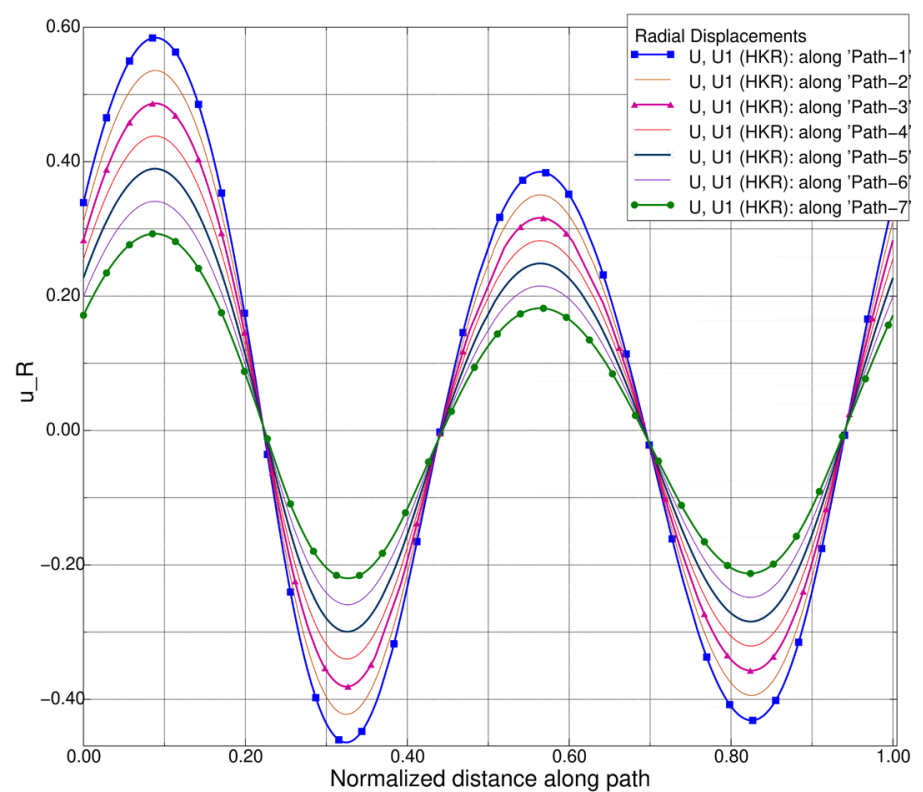

Figure 8. Normal drum displacement field generated by contact tractions. 
The normalized distance along the drum surface is specified by the formula $s_{\text {norm }}=s / 2 \pi R_{0}$. The deformed body is demonstrated in Figure 9. It is interesting to note that between the contact zones the normal displacement is negative, that is the surface moves inward. The normal displacement along the axial direction $y$ is not uniform, in the first section $y=1$ taking the largest value. If we separately draw the normal displacement only over the contact zones, we see, that in the boundary regions the normal displacement has a negative value (see Figure 10). This demonstrates that the wear is present in these domains.

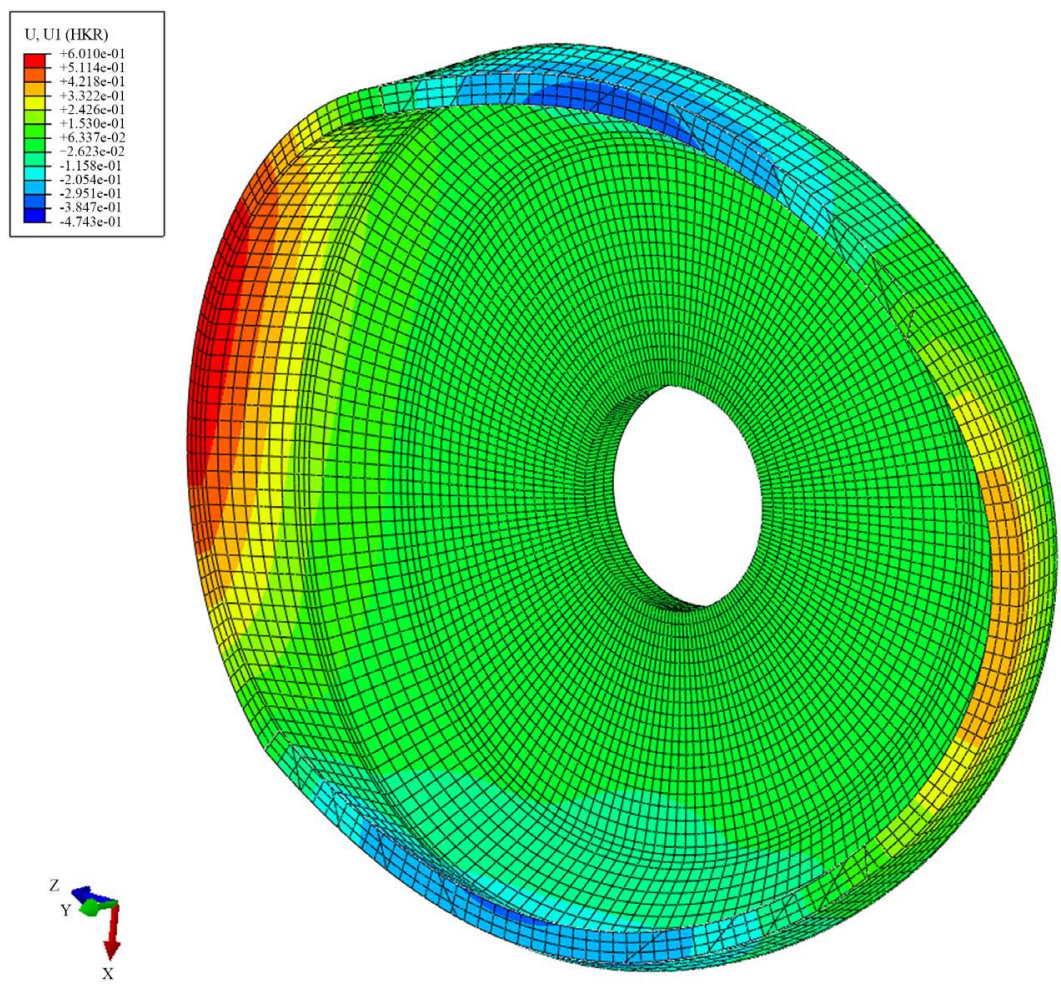

Figure 9. Drum in the loading state. The enlargement factor for displacement is 100.

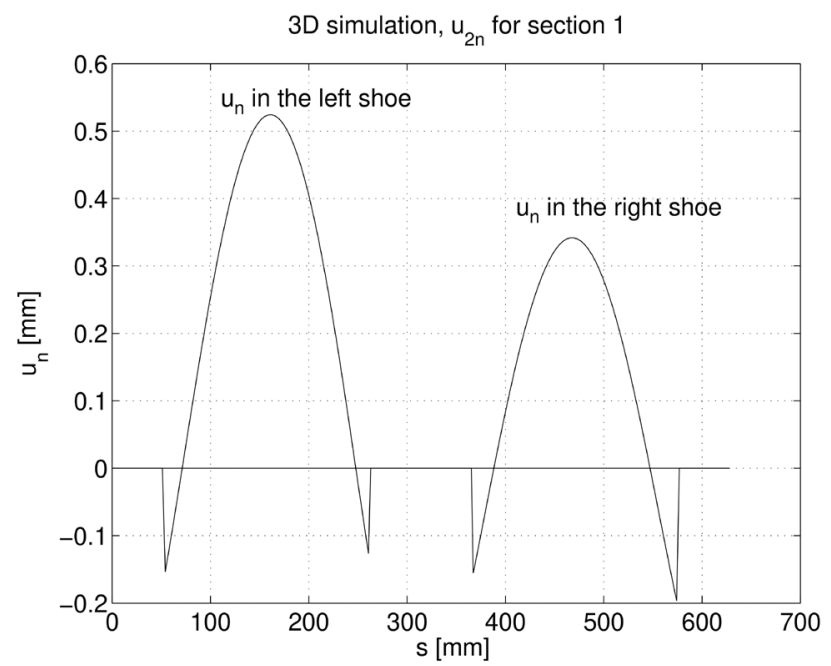

Figure 10. Normal displacement over the shoes in the steady wear state. 
For wear calculation, a simplified model is set up. The shoes are assumed as rigid, the elastic deformation is only in the brake drum. We give different rotation values for shoes, $\lambda_{l}$ for the left shoe, $\lambda_{r}$ for the right shoe. It is assumed that $\lambda_{r}=\lambda_{l} u_{n, \max , \text { right }} / u_{n, \max , \text { left }}$. In the steady wear state along the whole domain ( $\alpha_{i} \leq \alpha \leq \alpha_{u}, R_{0}$ ) there is contact, so the gap between the bodies is absent, and we can write geometrical Signorini contact condition

$$
d=u_{n}-u_{R}+w_{n}=0,
$$

where $u_{n}$ is the normal displacement of the brake drum in direction of the radius of cylindrical system, $u_{R}$ is the normal displacement from rigid body rotation of the shoe and $w_{n}$ denotes the wear value. We have

$$
u_{R}^{-}=\lambda_{M}^{-}\left[\left(R_{0} \cos \alpha^{-}+l_{z}\right) \sin \alpha^{-}-\left(R_{0} \sin \alpha^{-}-l_{x}\right) \cos \alpha^{-}\right] \quad(-\Rightarrow+)
$$

where $\alpha^{-}=\pi-\phi, \alpha^{+}=-\pi+\phi, \phi=s / R_{0}$, next - is used to denote the left shoe region, and + denotes the right shoe region. From Equation (23) it is easy to determine the shoe wear. The wear profiles are presented in Figure 11 for different values of rotations $\lambda_{l}$ and $\lambda_{r}$. Figure 11 is made by MATLAB system [12] without the color bar.

We take different $y$ sections for demonstration of the wear distribution (see Figure 12). Because the ring is elastic and at $y=1$ has the largest radial displacement, the wear will be smallest in this section. In these diagrams we take 3 different rigid body rotation values $\lambda_{l}$. It is seen that small value of $\lambda_{l}$ does not correspond to steady state. In the steady wear state the wear velocities $\dot{\lambda}_{M}^{\mp}$ are proportional to pressure (see Equations (15)-(19)) and the varying wear form is also proportional to pressure. Figure 13 demonstrates this fact, where the wear distribution curves translate nearly as rigid lines in the vertical direction for increasing wear rotation values. After reaching $\lambda_{l}=0.028$, the form of wear shape does not significantly vary, so the steady wear state has been reached.

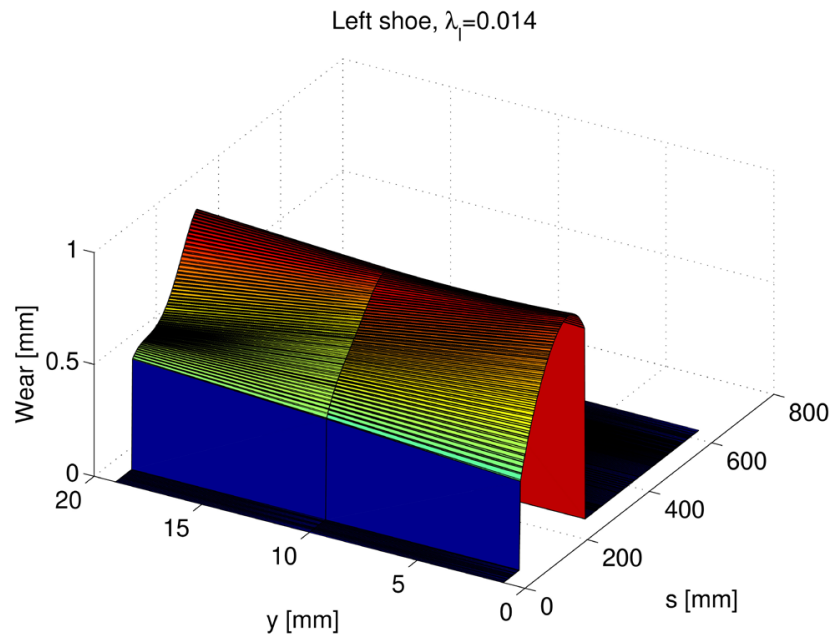

(a) 


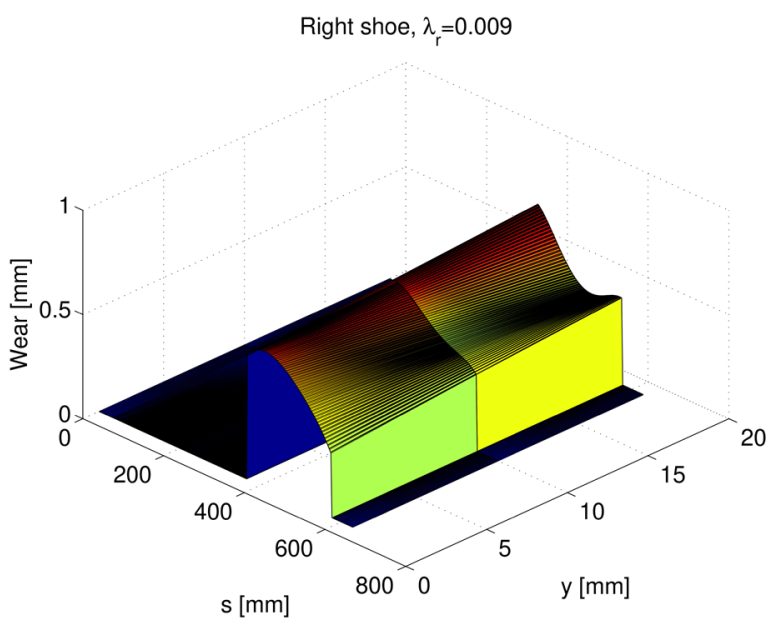

(b)

Left shoe, $\lambda_{1}=0.028$

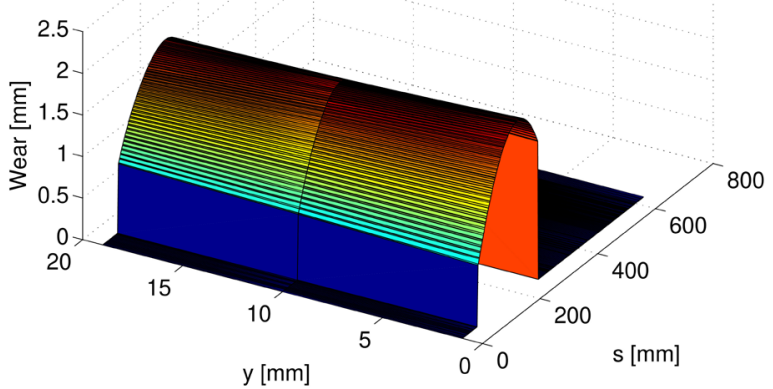

(c)

Right shoe, $\lambda_{\mathrm{r}}=0.018$

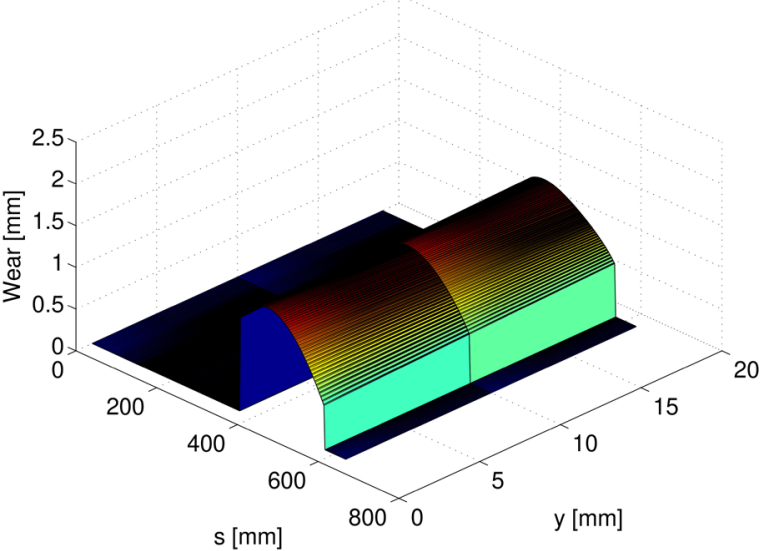

(d)

Figure 11. Wear at different shoes positions. (a) Wear over left shoe, $\lambda_{l}=0.014$, (b) Wear over right shoe, $\lambda_{r}=0.014 \frac{0.3417}{0.5241}=0.009$, (c) Wear over left shoe, $\lambda_{l}=0.028,(\mathrm{~d})$ Wear over right shoe, $\lambda_{r}=0.028 \frac{0.3417}{0.5241}=0.018$. 


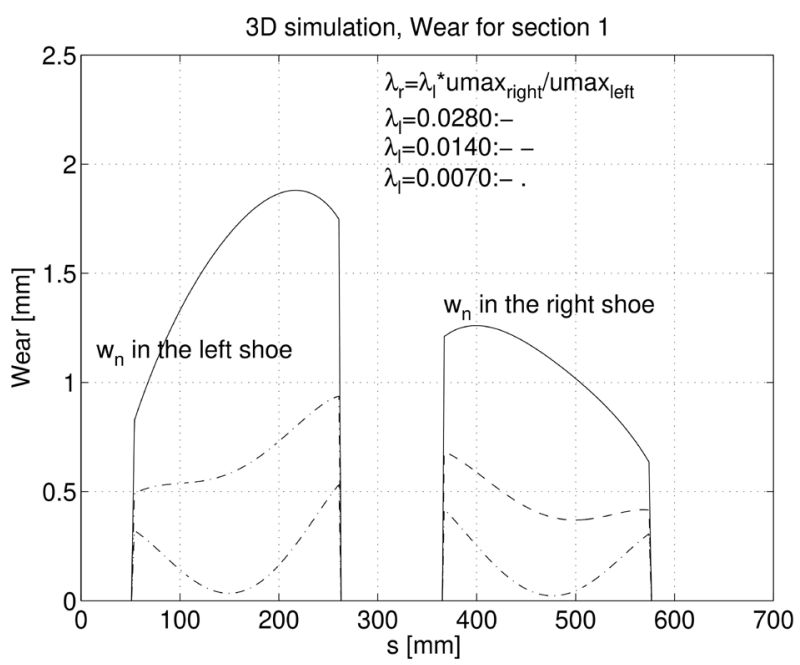

(a)

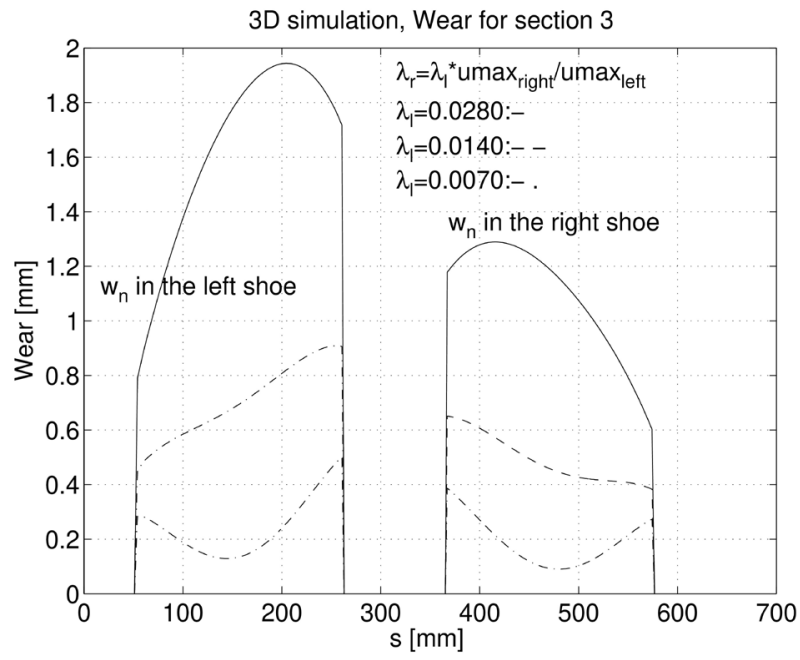

(b)

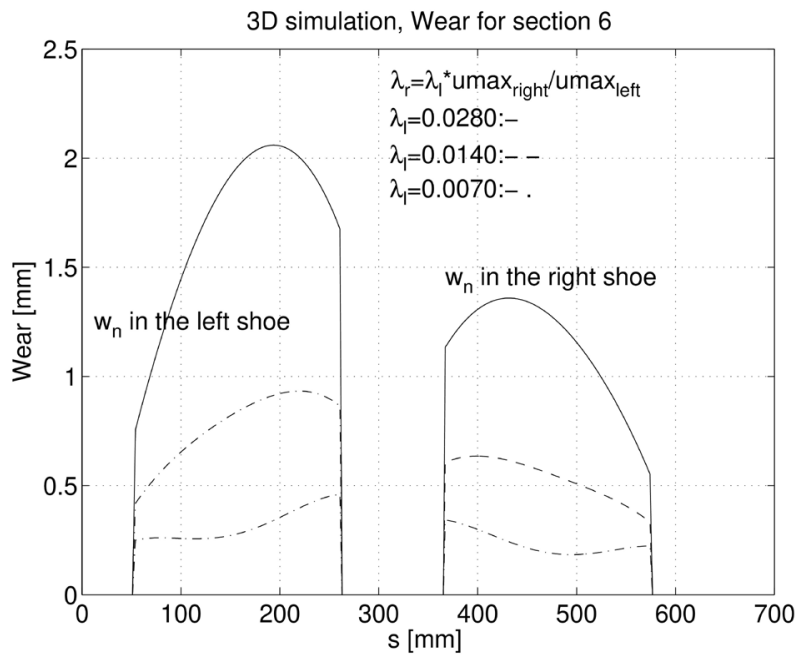

(c)

Figure 12. Wear of the shoes in different $y$ sections. (a) section $y=1$, (b) section $y=3$, (c) section $y=6$. 


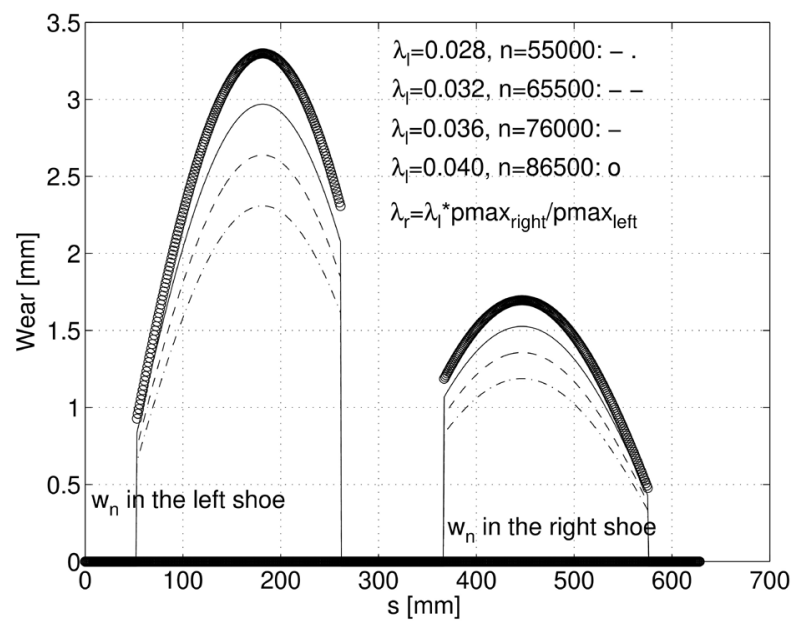

Figure 13. Wear profile evolution at increasing number of revolutions $n$ of the drum in the steady wear state.

\subsection{Estimation of the Steady Wear State Period}

Taking the assumed wear parameters, the values $a_{i}=b=1, q=1$, friction coefficient, angular velocity of the brake drum, distance $L$, load $F_{0}$, the calculated integrals (19) are $I_{D_{w}}^{-}=132 \times 10^{5}\left[\mathrm{~mm}^{4}\right], I_{D_{w}}^{+}=256 \times 10^{5}\left[\mathrm{~mm}^{4}\right]$ and the rigid body wear rotation velocities are $\dot{\lambda}_{M}^{-}=1.51 \times 10^{-7}[\mathrm{rad} / \mathrm{s}]$, $\dot{\lambda}_{M}^{+}=7.81 \times 10^{-8}[\mathrm{rad} / \mathrm{s}]$.

We take rotation of the left shoe $\lambda_{l}=0.028$. Supposing that wear rate is changing approximately in proportion to quadratic law in time and the initial value is equal to $3 \dot{\lambda}_{M}^{-}=4.53 \times 10^{-7}$, (this can be calculated from initial contact pressure), then $\dot{\lambda}^{-}=\dot{\lambda}_{M}^{-}\left(1+2 \frac{\left(\tau-T_{c}\right)^{2}}{T_{c}^{2}}\right)$. After time integration we find $T_{c}=\frac{3}{5} \frac{\lambda_{M}^{-}}{\dot{\lambda}_{M}^{-}}=30.91 \mathrm{~h}$. For the right shoe is $\lambda_{r}=0.018=\lambda_{M}^{+}, \dot{\lambda}_{M}^{+}=7.81 \times 10^{-8}$, then $T_{c}=\frac{3}{5} \frac{\lambda_{M}^{+}}{\dot{\lambda}_{M}^{+}}=38.41 \mathrm{~h}$. It is clear that the time period for the right shoe is larger than for the left shoe, because the wear rate is smaller. As in the steady wear state $\dot{\lambda}_{M}^{-}=$const, the increment of the shoe rotation angle is $\Delta \lambda=\dot{\lambda}_{M}^{-}\left(T-T_{c}\left(\lambda_{l}=0.028\right)\right)$. From this fact we find that $T\left(\lambda_{l}=0.032\right)=45.77 \mathrm{~h}, \quad T\left(\lambda_{l}=0.036\right)=53.13 \mathrm{~h}, \quad T\left(\lambda_{l}=0.040\right)=60.49 \mathrm{~h}$. Knowing angular velocity $\omega=2.5[1 / \mathrm{sec}]$, it is easy to calculate the number of revolutions of the drum $n=3600 T \omega / 2 \pi, n\left(\lambda_{l}=0.032\right)=65500$, etc.

\section{Conclusion}

For a braking system in the steady wear state, the contact pressure distribution provides a good possibility to find the maximum brake moment in the optimal design problem. This distribution can be determined from the minimization procedure of the wear dissipation power. The wear of the shoes can be easily 
calculated using the steady contact pressure distribution. In our analysis, the shoe was supposed as rigid body; the drum was assumed as linear elastic; then the wear rate is overestimated. The numerical predictions presented in the paper require experimental verification.

\section{Acknowledgements}

The present research was partially supported by the Hungarian Academy of Sciences, by the grant National Research, Development and Innovation Office-NKFIH: K115701.

\section{Conflicts of Interest}

The authors declare no conflicts of interest regarding the publication of this paper.

\section{References}

[1] Páczelt, I. and Mróz, Z. (2009) On the Analysis of Steady Sliding Wear Process. Tribology International, 42, 275-283. https://doi.org/10.1016/j.triboint.2008.06.007

[2] Koessler, P. (1957) Berechnung von Innenbacken-Bremsen für Kraftfahrzeuge. Stuttgart, Franckh'sche.

[3] Rao, S.S. and Cao, L.T. (2002) Optimum Design of Mechanical Systems Involving Internal Parameters. Journal of Mechanical Design, 124, 465-472. https://doi.org/10.1115/1.1479691

[4] Huang, Y.M. and Shyr, J.S. (2002) On Pressure Distributions of Drum Brakes. Journal of Mechanical Design, 124, 115-120. https://doi.org/10.1115/1.1427694

[5] Hohmann, C., Schiffer, K., Oerter, K. and Reese, H. (1999) Contact Analysis for Drum Brakes and Disk Brakes Using ADINA. Computers and Structures, 72, 185-198. https://doi.org/10.1016/S0045-7949(99)00007-3

[6] Watson, C. and Newcomb, T.P.A. (1990) Three-Dimensional Finite Approach to Drum Brake Analysis. Proceedings of Institution of Mechanical Engineering, 204, 93-102. https://doi.org/10.1243/PIME_PROC_1990_204_140_02

[7] Ahmed, I., Fatouh, Y. and Wael, A. (2014) A Parametric FE Modeling of Brake for Non-Linear Analysis. International Journal of Energy and Environment, 5, 97-110.

[8] Páczelt, I. and Mróz, Z. (2011) Numerical Analysis of Steady Thermo-Elastic Wear Regimes Induced by Translating and Rotating Punches. Computers and Structures, 89, 2495-2521. https://doi.org/10.1016/j.compstruc.2011.06.001

[9] Páczelt, I. and Mróz, Z. (2015) Analysis of Thermo-Mechanical Wear Problems for Reciprocal Punch Sliding. Advances in Engineering Software, 80, 139-155. https://doi.org/10.1016/j.advengsoft.2014.09.012

[10] Mróz, Z., Kucharski, S. and Páczelt, I. (2018) Anisotropic Friction and Wear Rules with Account for Contact State Evolution. Wear, 396-397, 1-11. https://doi.org/10.1016/j.wear.2017.11.004

[11] Banichuk, N.V. (2011) Introduction to Optimization of Structures. Springer, London.

[12] https://www.mathworks.com/products/matlab-online.html 